\title{
Immediate Effects of a Single Exercise on Behavior and Memory in the Early Period of Traumatic Brain Injury in Rats
}

\author{
Kyung Jae Yoon, $\mathrm{MD}, \mathrm{PhD}^{1,2}$, Dae Yul Kim, $\mathrm{MD}, \mathrm{PhD}^{3}$
}

\begin{abstract}
${ }^{1}$ Department of Physical and Rehabilitation Medicine, Kangbuk Samsung Hospital, Sungkyunkwan University, School of Medicine, Seoul; ${ }^{2}$ Medical Research Institute, Regenerative and Neuroscience Laboratory, Kangbuk Samsung Hospital, Sungkyunkwan University, School of Medicine, Seoul; ${ }^{3}$ Department of Rehabilitation Medicine, Asan Medical Center, University of Ulsan College of Medicine, Seoul, Korea
\end{abstract}

\begin{abstract}
Objective To evaluate the immediate effect of single exercise on physical performance and memory in the early stage of traumatic brain injury (TBI) in rats.

Methods Ninety TBI rats were randomly assigned to T0 (sedentary), T10 (treadmill $10 \mathrm{~m} / \mathrm{min}$ for 30 minutes), or T20 (treadmill $20 \mathrm{~m} / \mathrm{min}$ for 30 minutes) groups, on day 3 (D3), D7, and D14 after TBI, respectively. Rotarod (RR), Barnes maze (BM), brain magnetic resonance imaging (MRI) and MR spectroscopy were performed immediately before and 6 hours after exercise. Rats were sacrificed for immunohistochemistry with heat shock protein 70 (Hsp70) and glial fibrillary acidic protein (GFAP).

Results On D3, the T10 and T20 groups demonstrated significant improvement in RR ( $<<0.05)$. On D7, only the T20 group showed significantly enhanced RR ( $\mathrm{p}<0.05)$. In BM on D3, the T20 group showed significant deterioration compared with the other groups $(\mathrm{p}<0.05)$. Lesion volume did not significantly differ among the groups. MR spectroscopy on D3 showed that only the T20 group had significantly increased choline/creatine and $0.9 /$ creatine $(\mathrm{p}<0.05)$. In the perilesional area on D3, only T20 had a significantly higher Hsp70 and GFAP than the T0 group. On D7, Hsp70 was significantly higher in the T20 group than in the T0 group ( $\mathrm{p}<0.05)$. In the ipsilesional hippocampus on D3, the T20 group showed a significantly higher Hsp70 and GFAP than the T0 group $(\mathrm{p}<0.05)$.

Conclusion A single session of low-intensity exercise in the early period of TBI improves behavioral performance without inducing cognitive deficits. However, high-intensity exercise can exacerbate cognitive function in the early period after TBI. Therefore, the optimal timing of rehabilitation and exercise intensity are crucial in behavior and memory recovery after TBI.
\end{abstract}

Keywords Traumatic brain injuries, Exercise, Recovery, Memory, Rehabilitation

Received October 10, 2017; Accepted November 27, 2017

Corresponding author: Dae Yul Kim

Department of Rehabilitation Medicine, Asan Medical Center, University of Ulsan College of Medicine, 88 Olympic-ro 43-gil, Songpa-gu, Seoul 05505, Korea. Tel: +82-2-3010-3793, Fax: +82-2-3010-6964, E-mail: dykimmart@gmail.com

ORCID: Kyung Jae Yoon (http://orcid.org/0000-0002-2765-4309); Dae Yul Kim (http://orcid.org/0000-0003-1275-1736).

(c) This is an open-access article distributed under the terms of the Creative Commons Attribution Non-Commercial License (http://creativecommons.org/ licenses/by-nc/4.0) which permits unrestricted noncommercial use, distribution, and reproduction in any medium, provided the original work is properly cited. Copyright $\odot 2018$ by Korean Academy of Rehabilitation Medicine 


\section{INTRODUCTION}

Traumatic brain injury (TBI) is increasingly occurring in the industrialized world [1]. Additionally, with the growing elderly population, the incidence of geriatric TBI has also been increasing [2]. A recent study reported that the incidence of TBI has increased from 47.3 to 849 per 100,000 population per year [3]. Although TBI is a leading cause of disability, trials of neuroprotective treatments in the early injury period are very rare [4].

It has been shown that exercise has beneficial effects on behavior and memory in the healthy brain $[5,6]$. Previous studies have also reported that exercise enhances neural plasticity and elicits plasticity-associated molecules including glial fibrillary acidic protein (GFAP) [7-9]. The injured brain, however, may be vulnerable to excitation during the acute stage of TBI. Some reports have demonstrated that early exercise after TBI exacerbated brain damage and interfered with functional recovery $[10,11]$. Although many studies have reported that exercise could ameliorate the disabilities after TBI, the therapeutic time window for exercise during the early period of TBI remains controversial [12].

The benefit of regular exercise is usually derived from the accumulative effects of repeated sessions of a single exercise. However, identifying the immediate effect of a single session of exercise might provide basic neurophysiologic information, which will then help in the development of new treatment strategies for the early stage of TBI. In other studies, a single session of exercise in healthy persons was reported to promote motor performance and improve cognition [13,14]. However, no report has been presented on the effect of a single exercise on the behavior and memory recovery of an injured brain.

Therefore, the aim of the current study was to demonstrate the immediate effect of a single session of exercise on physical performance and memory function in the early stage of TBI.

\section{MATERIALS AND METHODS}

\section{TBI model}

Male Sprague-Dawley rats (250-300 g) were housed in cages under a controlled environment $\left(22.0^{\circ} \mathrm{C}-24.0^{\circ} \mathrm{C}\right)$, and maintained in a 12:12-hour light-dark cycle. All rats had free access to food and water. All experiments in this study were approved by the Asan Institute for Life Sciences, Asan Medical Center (No. 2010-14-203). The lateral fluid percussion method was used for generating the TBI model [15]. Rats were anesthetized with isoflurane (2\% isoflurane in a 1:2 mixture of $\mathrm{O}_{2} / \mathrm{N}_{2} \mathrm{O}$ ) via mask. A heating pad was applied to keep the body temperature at $37^{\circ} \mathrm{C} \pm 0.5^{\circ} \mathrm{C}$. The skull was prepared with a topical depilatory agent and betadine solution. A 3-mm craniectomy was created on the right side, $1.5 \mathrm{~mm}$ from the bregma, over the M1 position [16]. A modified Luer-Lock (model 211 B4; Kistler Instrumental Corp., Amherst, NY, USA) was positioned in the craniectomy site with methyl methacrylate. The saline-filled device was attached to the skull via the Luer-Lock, and a single moderate severity pulse (3.5-4.0 atm pressure) was applied with rapid injection of saline onto the craniectomized brain site.

\section{Treadmill exercise}

All rats were pretrained on a motorized treadmill at a speed of $5-15 \mathrm{~m} / \mathrm{min}$ for $10-20$ minutes, three times prior to the TBI operation. Rats of the exercise group ran on the treadmill with a $0^{\circ}$ inclination at a speed of $10 \mathrm{~m} / \mathrm{min}$ (T10) or $20 \mathrm{~m} / \mathrm{min}$ (T20) for 30 minutes on day 3 (D3), D7, and D14 after TBI. Rats of the sedentary group (T0) were placed on a stationary treadmill (without running) at the same time points.

\section{Behavioral and memory tests}

The rotarod (RR) test was performed to assess motor coordination. The rats were placed on a $\mathrm{RR}$, rotating at an initial speed of $4 \mathrm{rpm}$. The speed was accelerated to $40 \mathrm{rpm}$ over 4 minutes. The time taken for the rats to fall over was measured three times and averaged by a researcher blinded to the objective of the experiment. The Barnes maze (BM) test was applied to assess the spatial memory functions of the rats, which has a white circular platform (diameter, $120 \mathrm{~cm}$ ) containing 18 holes of 10 $\mathrm{cm}$ diameter in the periphery. A $20 \times 15 \times 20 \mathrm{~cm}^{3}$ black goal box was set below one of these 18 holes to test whether the rats can remember the position of the black goal box and avoid the 1,000 lx lightened platform. The rats were placed at the center of the platform and allowed to enter the goal box. They were pretrained three times before the TBI operation. The time taken to find the goal box was recorded three times and averaged in a blinded manner. 


\section{Brain MRI and MR spectroscopy}

Anesthesia induction was performed with an intraperitoneal injection of $1.0 \%$ ketamine in all TBI rats. The rats were then set in a 4.7-T Bruker BioSpec Imager (Bruker Medical System, Karlsruhe, Germany). Brain MRI and MR spectroscopy were applied by a blinded researcher according to a previously described method [17]. Briefly, a T2-weighted image (T2WI) sequence with 4,500 repetition times (ms) and 80 echo times (ms) was applied. The slice thickness was $1.5 \mathrm{~mm}$. The cross-sectional area was measured with ParaVision 3.0 software (Bruker Medical System). The lesion volume was calculated from the cross-sectional area and slice thickness.

To measure the metabolites with MR spectroscopy, the volume of interest was selected over the perilesional area with a cubic volume of $2 \times 2 \times 2 \mathrm{~mm}^{3}$ in the injured hemisphere in T2WI. Bruker's XWIN-NMR software was used to process all raw spectroscopic data. The metabolites were identified according to their chemical shift, as observed in the MR spectroscopy, in parts per million values: choline (Cho), 3.2; creatine (Cr), 3.03; N-acetylaspartate (NAA), 2.0; and lactate (Lac), 1.3; 0.9. The Cho, NAA, Lac, and 0.9 peak areas were quantitated as relative ratios to $\mathrm{Cr}$, which was the internal reference for each rat, to allow the comparison of metabolite levels over time and between different animals [18].

\section{Immunohistochemistry}

The rats were anesthetized with $1.0 \%$ ketamine via intraperitoneal injection and sacrificed with intracardiac perfusion with phosphate-buffered saline and $4.0 \%$ paraformaldehyde. The brains were sliced coronally at a $1.0-\mathrm{mm}$ thickness. The sliced brains were fixed with $10 \%$ formalin and embedded in paraffin wax for immunohistochemistry. A paraffin block was acquired from the perilesional area and ipsilesional hippocampus. Each block was sliced into $4-\mu \mathrm{m}$-thick sections and mounted on poly-L-lysine-coated slides. These slides were serially processed, including deparaffinization with xylene, dehydration with ethanol, saline washing, and microwaving in citrate buffer for 10 minutes. A 3\% hydrogen peroxide solution was used to block endogenous peroxidase activity for 30 minutes and reduce nonspecific immunoreactivity. Primary antibodies against heat shock protein 70 (Hsp70; Abcam, Cambridge, MA, USA) and GFAP (Abcam) were used to investigate the effects on neural plasticity. The expression of Hsp70 and GFAP could be detected around the perilesional area from 4 hours after injury [19]. The secondary antibody used was an anti-mouse horseradish peroxidase/3,3'-diaminobenzidine system (Labvision, Fremont, CA, USA). Six views of the perilesional area and the ipsilesional hippocampus (CA1, CA3, and dentate gyrus) were assessed and averaged in a blinded manner by a pathologist who counted the stained cells under a light microscope.

\section{Experimental design}

The TBI rats were randomly assigned to the T0, T10, and T20 groups (10 rats per group) on D3, D7, and D14 after TBI, respectively (a total of 90 rats). The TBI rats of each group were kept in cages until treadmill training. The rats in the T10 group performed a single session of treadmill exercise at a speed of $10 \mathrm{~m} / \mathrm{min}$ for 30 minutes in 1 day. Those in the T20 group performed a single session of treadmill exercise at a speed of $20 \mathrm{~m} / \mathrm{min}$ for 30 minutes. The rats in the T0 group were placed on a stationary treadmill for 30 minutes.

RR and BM tests were performed before treadmill exercise and 6 hours after the exercise in all rats. The ratios of $\mathrm{RR}$ and BM were calculated as follows:

$$
\frac{\text { Final data }}{\text { Baseline data }} \times 100 \text {. }
$$

Brain MRI and MR spectroscopy were performed immediately before treadmill exercise and 6 hours after exercise in all rats. The ratios of lesion volume and metabolites were obtained as follows:

$$
\frac{\text { Data of } 6 \text { hours after exercise }}{\text { Baseline data }} \times 100 \text {. }
$$

After all evaluations, the rats were sacrificed on the day of each evaluation for immunohistochemistry.

\section{Statistical analysis}

PASW version 24.0 software was used for all statistical analyses. One-way analysis of variance (ANOVA) or the Kruskal-Wallis test was performed to evaluate the differences between groups (T0, T10, and T20) for each period after TBI (D3, D7, and D14) for the RR, BM, lesion volume, MR spectroscopy metabolites, and immunohistochemistry data (Hsp70 and GFAP). A p-value less than 0.05 was considered significant in one-way ANOVA. If a significant difference was found among groups in a one- 
way ANOVA, further analyses were conducted using the Scheffe post-hoc test and Bonferroni correction to evaluate the differences among the three groups, whereby a pvalue less than 0.0167 was considered significant.

\section{RESULTS}

\section{Behavior and memory}

The baseline results of the RR and BM tests were not significantly different among the three groups on D3, D7, and D14 ( $>>0.05$ ) (Table 1). Among the D3 groups, $\mathrm{T} 10$ and $\mathrm{T} 20$ showed significant benefit in the RR ratio $(\mathrm{p}<0.05)$ (Fig. 1A). On D7, however, only T20 showed significantly higher RR ratios than the other $\mathrm{D} 7$ groups $(\mathrm{p}<0.05)$ (Fig. 1A). No significant difference was found among the three groups on D14 ( $>0.05)$. In the BM test, the T20 group showed significantly higher BM ratios than the other groups on D3, which indicates deterioration $(\mathrm{p}<0.05)$ (Fig. 1B). On D7 and D14, no significant differences in the BM ratios were found among the three groups ( $\mathrm{p}>0.05)$.

\section{Lesion volume and brain metabolites}

The baseline results of the volume of injured brain showed no significant difference among the three groups on D3, D7, and D14 ( $>>0.05)$ (Table 1). The ratios of volume of injured brain were not significantly different among the groups on D3, D7, and D14 ( $>$ >0.05) (Fig. 2A).

The baseline data of Cho/Cr, NAA/Cr, Lac/Cr, and $0.9 / \mathrm{Cr}$ were not significantly different among the three groups on D3, D7, and D14 (p>0.05) (Table 1). Among the D3 groups, a significant increase in $\mathrm{Cho} / \mathrm{Cr}$ and $0.9 / \mathrm{Cr}$ at 6-hour post-exercise was only shown in the T20 group $(\mathrm{p}<0.05)$ (Fig. 2B, 2C). No significant difference was shown among the three groups on D7 and D14 ( $>0.05)$.

\section{Immunohistochemistry}

In the perilesional area, only the T20 group showed significantly higher numbers of Hsp70- and GFAP-stained cells than the T0 group on D3 ( $\mathrm{p}<0.05$ ) (Fig. 3A, 3B). On D7, the number of Hsp70-stained cells was significantly higher in the T20 group than in the T0 group $(\mathrm{p}<0.05)$ (Fig. $3 \mathrm{~A})$.

In the ipsilesional hippocampus, only the T20 group showed significantly higher numbers of Hsp70- and GFAP-stained cells than the T0 group on D3 ( $\mathrm{p}<0.05)$ (Fig.

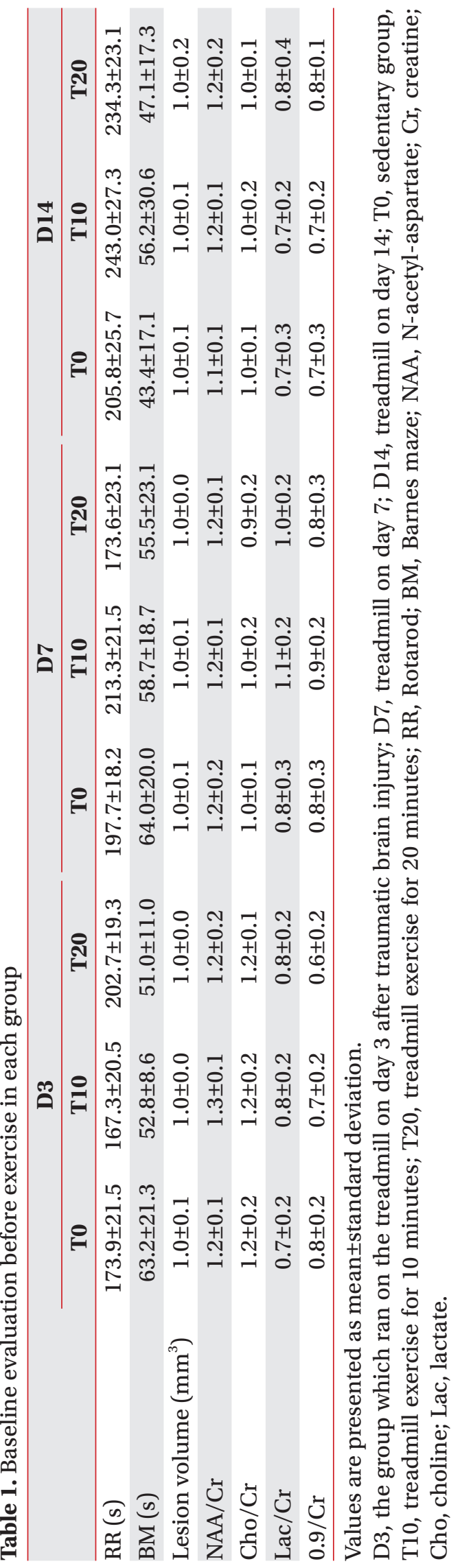


A

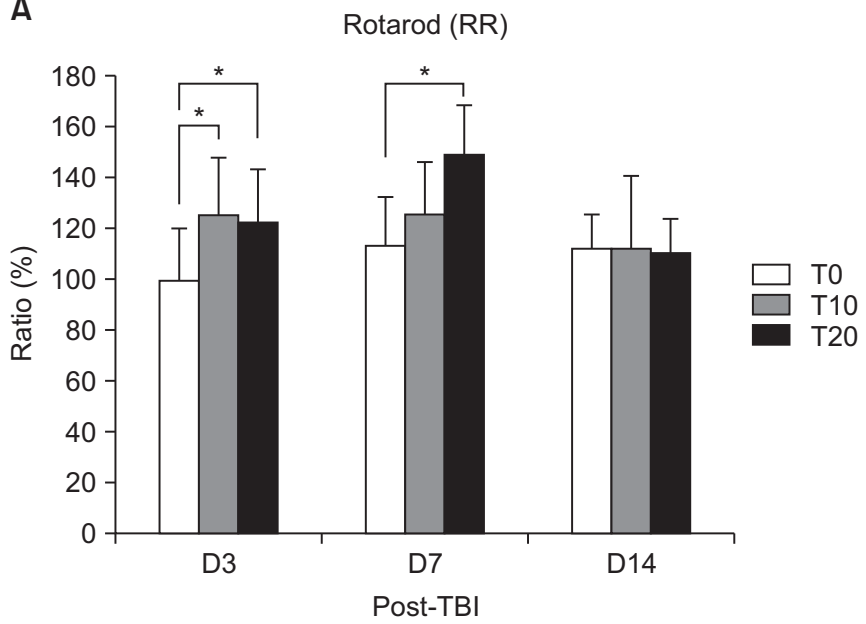

B

Barnes maze (BM)

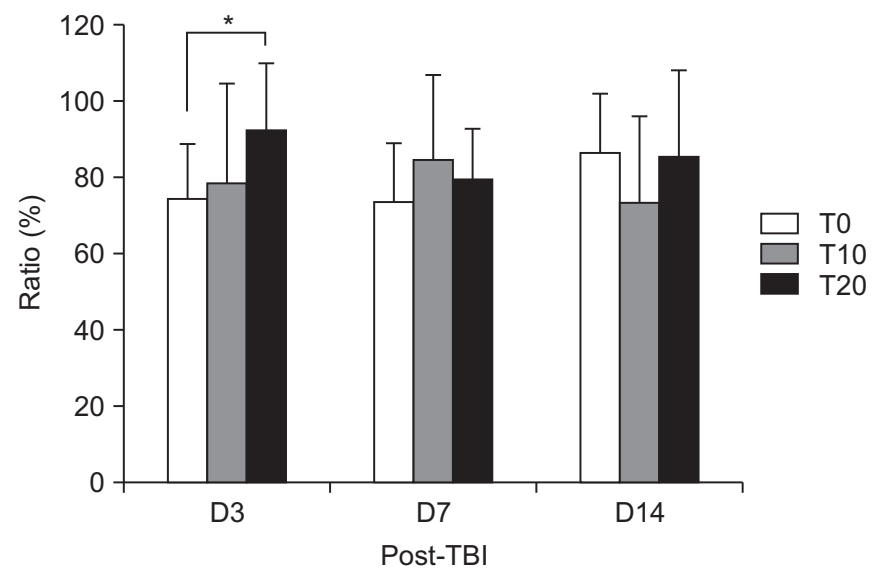

Fig. 1. The results of the Rotarod (RR) and Barnes maze (BM) tests. (A) In RR test, the T10 and T20 groups showed significantly higher RR ratios compared with T0 among the D3 groups. On D7, however, only the T20 group showed significantly higher RR ratios than the other groups. No significant difference was found among the three groups on D14. (B) In BM test, the T20 group showed significantly lower BM ratios than the other groups on D3. On D7 and D14, no significant differences were found in the BM ratios among the three groups. TBI, traumatic brain injury. ${ }^{*} \mathrm{p}<0.0167$, one-way ANOVA with the Scheffe post-hoc analysis.

3C, 3D). On D7 and D14, no significant difference was shown in the number of Hsp70- and GFAP-stained cells among the three groups $(\mathrm{p}>0.05)$.

\section{DISCUSSION}

The present results showed that a single session of exercise with a high intensity (T20) had an immediate effect on behavioral function in the earlier period of TBI (D3). However, cognitive deterioration was also observed in the T20 group. Interestingly, low-intensity exercise (T10) in the same period (D3) had a benefit on behavioral performance without inducing cognitive deficits. In addition, the high-intensity exercise group (T20) also showed motor improvement on D7 without cognitive exacerbation. However, a delayed single exercise (D14) did not have any immediate effect on motor and memory function. These findings suggest that a single session of exercise has different effects on behavior and memory according to the intensity of the exercise and the time window after TBI.

After TBI, the surviving cells are in a state of energy crisis and are vulnerable to secondary stimulation [20]. Therefore, premature exercise after TBI may reduce the capacity for plasticity of the brain $[10,11]$. In previous studies, however, the exercise intervention consisted of repeated sessions of a single exercise. No study has evaluated the effect of a single session of exercise on the injured brain. In this study, we showed that low- and high-intensity exercise at 3 days after TBI (D3) can help motor recovery from an induced TBI. However, the rats subjected to high-intensity exercise had more cognitive impairment than the control and the low-intensity exercise groups.

The mechanism of this different effects is unclear. Since increased body temperature can cause neurological injury [21], we suggest that increases in body temperature induced by intense exercise may adversely affect the recovery from TBI through a similar mechanism. At the cellular level, intense stress and exercise are known to activate the secretion of glucocorticoids [22], and endogenous glucocorticoid contributes to the basal level of brain injury resulting from cerebral ischemia [23]. This may underlie the adverse effects of intense exercise observed in this study.

Intense exercise also inhibits the expression of Bcl-2, and this may have a role in cognitive impairment after intense exercise [24]. In the early phase of TBI, cells around the injured area tend to react more sensitively to excitatory transmitters. Thus, early induction of intense exercise after brain injury may cause excessive secretion of excitatory neurotransmitters including glutamate or catecholamine [25], increased nerve impulses induced 
A

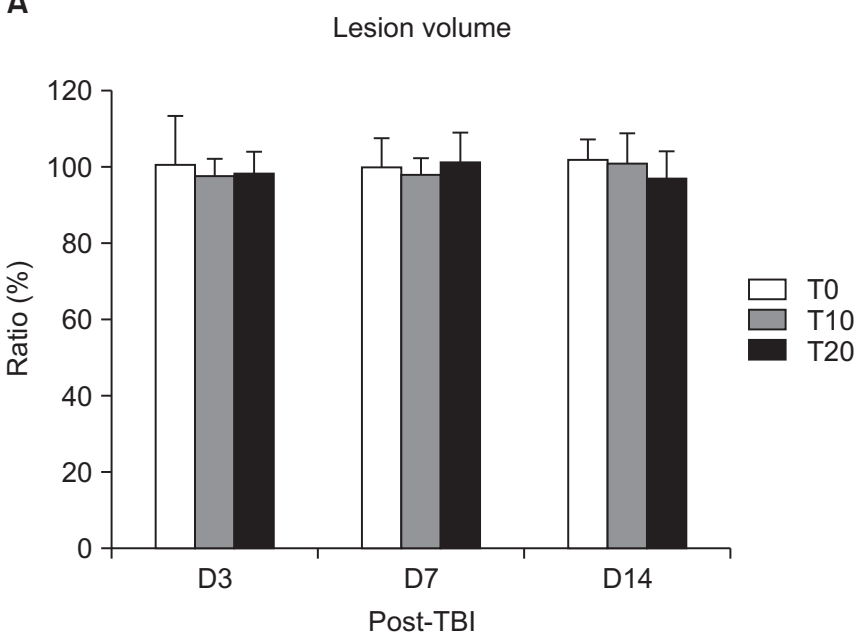

C

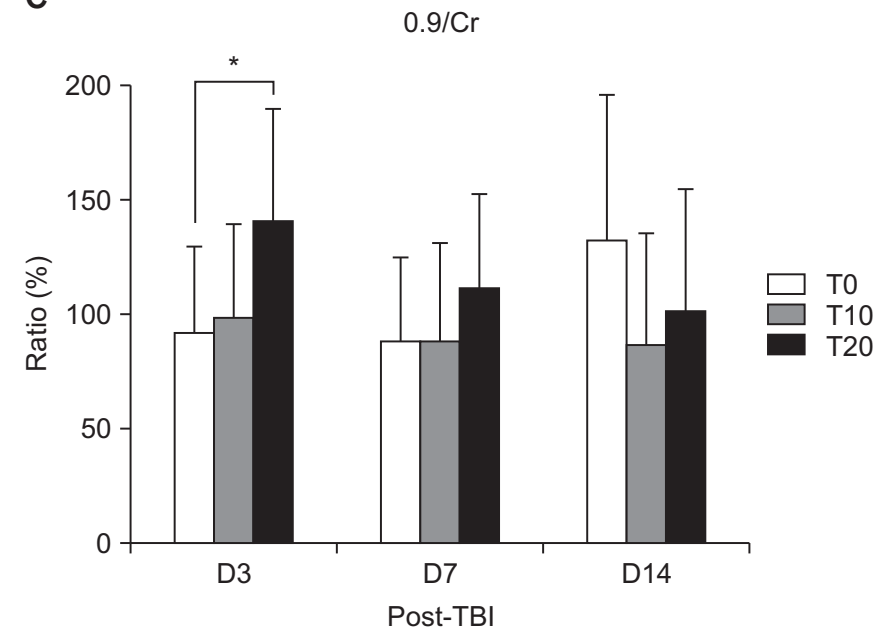

by the N-methyl-d-aspartate receptor, and fewer gammaaminobutyric acid-induced inhibitive reactions [26].

Our results demonstrated that high-intensity exercise on a treadmill at 7 days after TBI (D7) does not attenuate cognitive deficits. This result suggested that high-intensity treadmill exercise is not harmful for cognitive function around that period after TBI. Previous research, however, has shown that wheel running resulted in impaired cognitive performance in the early phase after TBI [27]. That result suggested that the intensity of exercise is a crucial factor in the early phase after TBI. Treadmill exercise has been suggested to affect multiple aspects of the physiological system, including the endocrine and immune systems, with elevated corticosterone, altered inflammatory cytokine expression, or immunosuppression [28]. Elevated levels of corticosterone have been demonstrated to negatively affect hippocampal plasticity [29]. Brain-
B

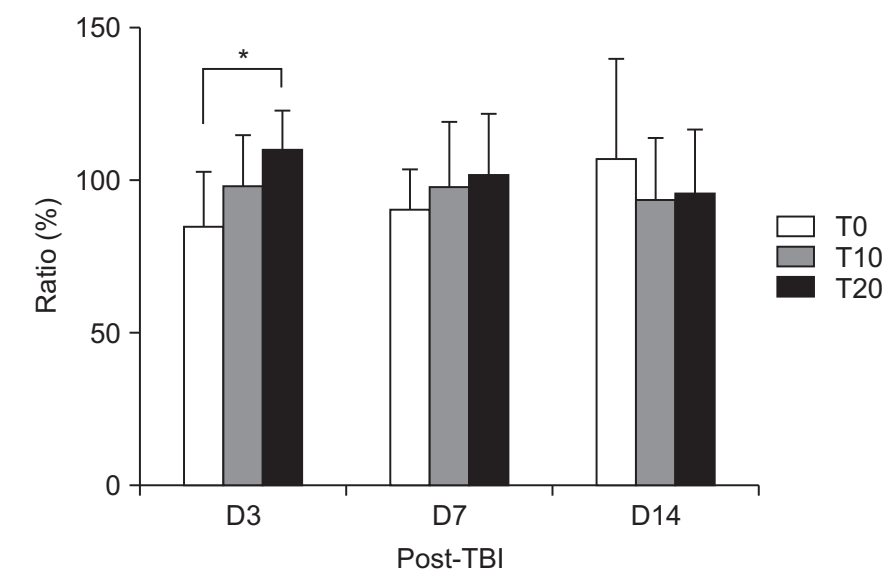

Fig. 2. The results of the volume of injured brain, Cho/Cr, and $0.9 / \mathrm{Cr}$. (A) Results of lesion volume measured by MRI showed no significant difference among the groups on D3, $\mathrm{D} 7$, and D14. Among the D3 groups, $\mathrm{Cho} / \mathrm{Cr}$ (B) and 0.9/Cr (C) measured by MR spectroscopy increased significantly in the T20 group. No significant differences were found among the three groups on D7 and D14. Cho, choline; Cr, creatine; TBI, traumatic brain injury. ${ }^{*} \mathrm{p}<0.0167$, one-way ANOVA with the Scheffe post-hoc analysis.

derived neurotrophic factor expression can be downregulated by circulating glucocorticoids at the mRNA and protein levels [30]. Based on these studies, it is possible that high-intensity treadmill exercise after the early phase of TBI may have been implemented as a stressor. Regardless of exercise intensity, treadmill exercise at 14 days after TBI (D14) does not affect motor recovery and cognitive function. These results suggested that the optimal timing of rehabilitation is important in motor recovery and cognition. However, the application of two different intensities is insufficient to firmly establish the optimal beginning of exercise after TBI; it is therefore difficult to conclude that cognitive deficits cannot be attenuated after the early phase in TBI. Further studies that include variable intensities after TBI will allow a more accurate and reliable assessment.

Cho and NAA measured by $1 \mathrm{H}$ MR spectroscopy are 
A
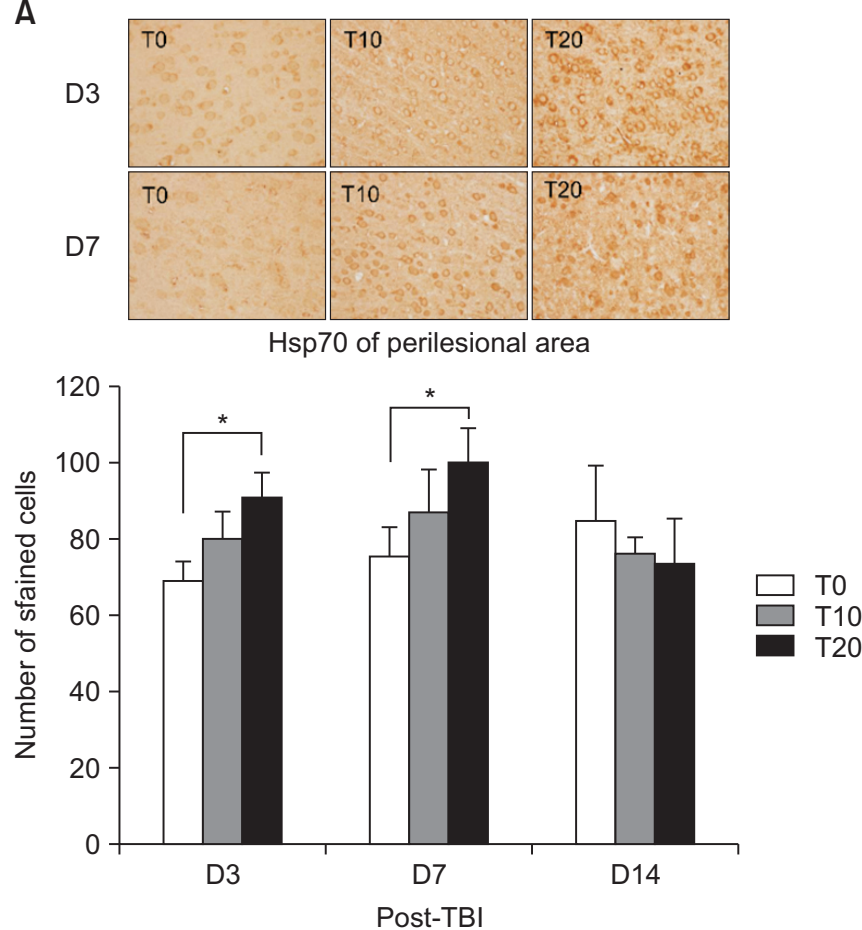

C

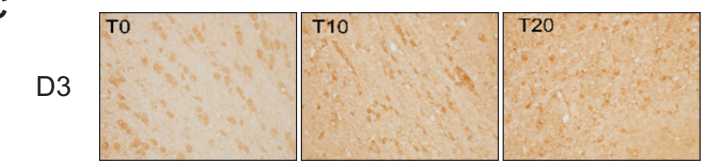

Hsp70 of ipsilesional hippocampus

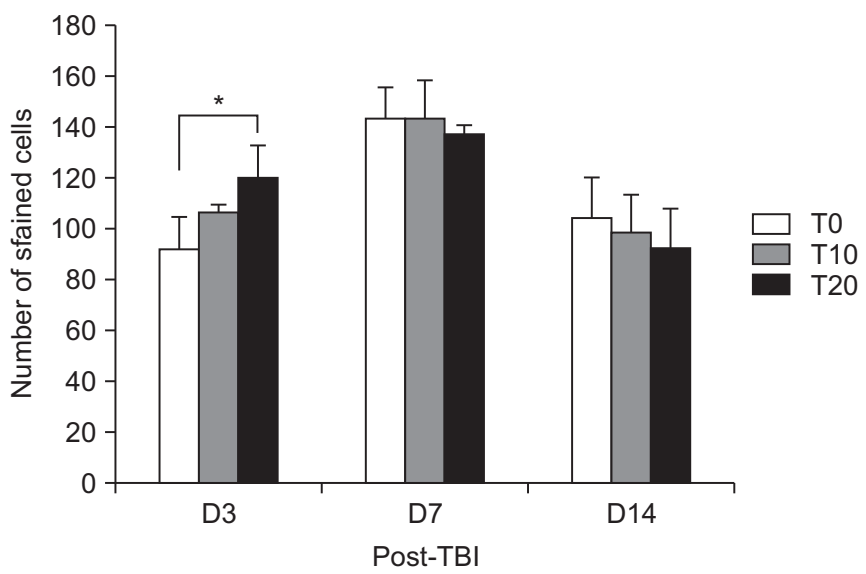

B
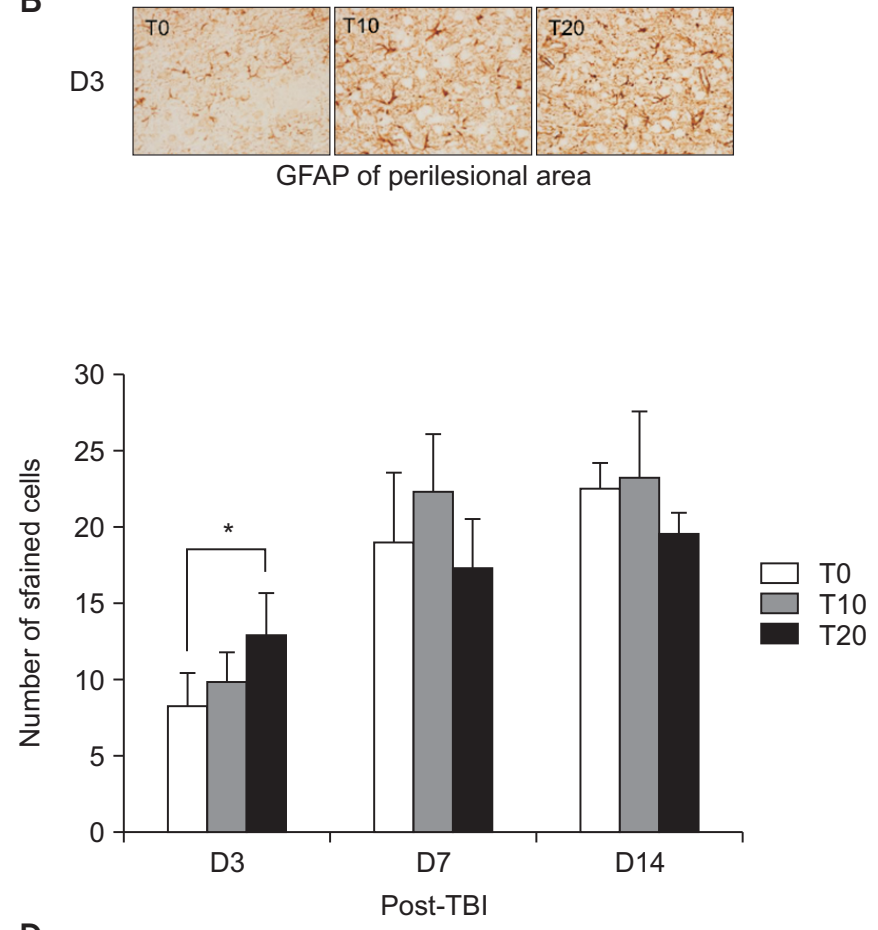

D
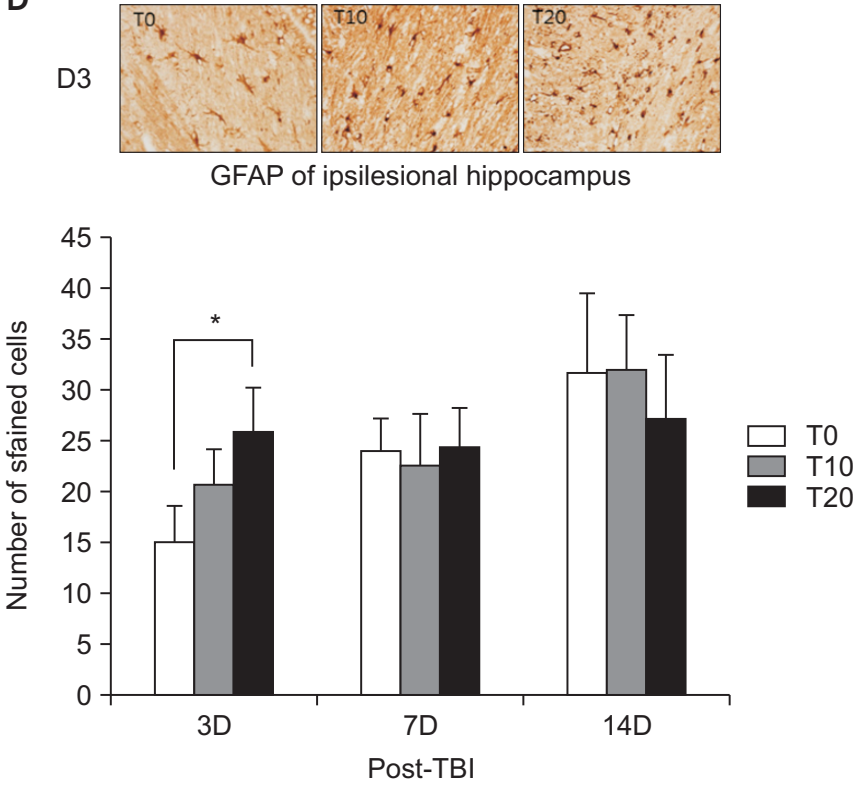

Fig. 3. Results of immunohistochemistry staining in perilesional area. The T20 group had significantly higher numbers of Hsp70-stained cells (A) and GFAP-stained cells (B) than the T0 group among the D3 groups in the perilesional area. On D7, the number of Hsp70-stained cells was significantly higher in the T20 group than in the T0 group (A). On D14, no significant difference was shown among the groups. The T20 group showed significantly higher numbers of Hsp70stained cells (C) and GFAP-stained cells (D) than the T0 group among the D3 groups in the ipsilesional hippocampus. On D7 and D14, no significant difference among the groups was observed. Hsp70, heat shock protein 70; GFAP, glial fibrillary acidic protein; TBI, traumatic brain injury. ${ }^{*} \mathrm{p}<0.0167$, one-way ANOVA with the Scheffe post-hoc analysis.

the structural component of cellular membrane and a predecessor of brain lipids, respectively. In states of neuronal damage, including TBI, the Cho level is expected to increase with a decrease of the NAA level. In this study, only T20 had significantly increased Cho/Cr. Moreover, astrocyte reactions accelerate the secretion of glutamate, 
which causes scarring around the cerebral infarction regions, resulting in adverse neurological effects. In the perilesional and ipsilesional hippocampal areas on D3, only T20 had significantly higher GFAP than T0. These findings meant that high intensity exercise has a neutral effect on cognitive deterioration in the early stage of TBI.

However, considering the role of Hsp70 on the protective effects against TBI, high expression of Hsp70 in the perilesional and ipsilesional hippocampal areas on D3 by high intensity exercise is difficult to interpret. It is possible that Hsp70 affects the motor recovery of TBI.

Our study has several limitations. First, we determined exercise intensity by calculating the percentage of maximum exercise velocity tolerated by rats. Our study would have been strengthened by using objective measures to quantify the varying degrees of exercise intensity, such as oxygen consumption and heart rate. Second, an important limitation of this study was the small sample size. Nevertheless, we were able to show the different effects of treadmill exercise after TBI in terms of intensity and start of exercise. The trend of the changes we observed warrants further study with a larger sample size to allow for a more discerning statistical analysis. Third, the rats were not familiarized with treadmill training before TBI induction. They only performed familiarized exercise 24 hours after TBI. Fourth, we could not include normal rats without TBI. The change in terms of exercise intensity and timing should have been compared. The immediate effects of treadmill exercise with a single intervention might not coincide with the effects of longer treadmill training. A longer exercise protocol needs to be applied to observe the changes.

In conclusion, low-intensity exercise in the early period of TBI enhanced behavioral performance without inducing cognitive deficits. A single session of exercise with high intensity in the early period after TBI could exacerbate cognitive function despite improvement in motor recovery. On the contrary, high-intensity exercise in the immediate period after TBI might be effective in motor recovery without cognitive decline. However, late treadmill training does not improve motor function. Our study suggests that exercise intensity and the optimal beginning of rehabilitation after TBI are crucial factors in motor recovery.

\section{CONFLICT OF INTEREST}

No potential conflict of interest was associated with this study.

\section{ACKNOWLEDGMENTS}

This study was supported and funded by the Asan Institute for Life Science (Task No. 2010-454) and by the Medical Research Funds from Kangbuk Samsung Hospital, Seoul, Korea.

\section{REFERENCES}

1. Hyder AA, Wunderlich CA, Puvanachandra P, Gururaj G, Kobusingye OC. The impact of traumatic brain injuries: a global perspective. NeuroRehabilitation 2007; 22:341-53.

2. Ramanathan DM, McWilliams N, Schatz P, Hillary FG. Epidemiological shifts in elderly traumatic brain injury: 18-year trends in Pennsylvania. J Neurotrauma 2012;29:1371-8.

3. Brazinova A, Rehorcikova V, Taylor MS, Buckova V, Majdan M, Psota M, et al. Epidemiology of traumatic brain injury in Europe: a living systematic Review. J Neurotrauma 2016 Aug 25 [Epub]. http://10.1089/ neu.2015.4126.

4. Menon DK, Maas AI. Traumatic brain injury in 2014: progress, failures and new approaches for TBI research. Nat Rev Neurol 2015;11:71-2.

5. Swain RA, Harris AB, Wiener EC, Dutka MV, Morris $\mathrm{HD}$, Theien BE, et al. Prolonged exercise induces angiogenesis and increases cerebral blood volume in primary motor cortex of the rat. Neuroscience 2003;117:1037-46.

6. van Praag H, Kempermann G, Gage FH. Running increases cell proliferation and neurogenesis in the adult mouse dentate gyrus. Nat Neurosci 1999;2:266-70.

7. Bernardi C, Tramontina AC, Nardin P, Biasibetti R, Costa AP, Vizueti AF, et al. Treadmill exercise induces hippocampal astroglial alterations in rats. Neural Plast 2013;2013:709732.

8. Saur L, Baptista PP, de Senna PN, Paim MF, do Nascimento P, Ilha J, et al. Physical exercise increases GFAP expression and induces morphological changes in hippocampal astrocytes. Brain Struct Funct 2014; 
219:293-302.

9. Keeler BE, Liu G, Siegfried RN, Zhukareva V, Murray M, Houle JD. Acute and prolonged hindlimb exercise elicits different gene expression in motoneurons than sensory neurons after spinal cord injury. Brain Res 2012;1438:8-21.

10. Kozlowski DA, James DC, Schallert T. Use-dependent exaggeration of neuronal injury after unilateral sensorimotor cortex lesions. J Neurosci 1996;16:4776-86.

11. Humm JL, Kozlowski DA, James DC, Gotts JE, Schallert T. Use-dependent exacerbation of brain damage occurs during an early post-lesion vulnerable period. Brain Res 1998;783:286-92.

12. Griesbach GS. Exercise after traumatic brain injury: is it a double-edged sword? PM R 2011;3(6 Suppl 1):S64-72.

13. Chang YK, Labban JD, Gapin JI, Etnier JL. The effects of acute exercise on cognitive performance: a metaanalysis. Brain Res 2012;1453:87-101.

14. McDonnell MN, Buckley JD, Opie GM, Ridding MC, Semmler JG. A single bout of aerobic exercise promotes motor cortical neuroplasticity. J Appl Physiol (1985) 2013;114:1174-82.

15. McIntosh TK, Vink R, Noble L, Yamakami I, Fernyak $\mathrm{S}$, Soares $\mathrm{H}$, et al. Traumatic brain injury in the rat: characterization of a lateral fluid-percussion model. Neuroscience 1989;28:233-44.

16. Fonoff ET, Pereira JF Jr, Camargo LV, Dale CS, Pagano RL, Ballester G, et al. Functional mapping of the motor cortex of the rat using transdural electrical stimulation. Behav Brain Res 2009;202:138-41.

17. Yoon KJ, Oh BM, Kim DY. Functional improvement and neuroplastic effects of anodal transcranial direct current stimulation (tDCS) delivered 1 day vs. 1 week after cerebral ischemia in rats. Brain Res 2012;1452:61-72.

18. Garnett MR, Blamire AM, Corkill RG, Cadoux-Hudson TA, Rajagopalan B, Styles P. Early proton magnetic resonance spectroscopy in normal-appearing brain correlates with outcome in patients following traumatic brain injury. Brain 2000;123(Pt 10):2046-54.

19. Li R, Fujitani N, Jia JT, Kimura H. Immunohistochemical indicators of early brain injury: an experimental study using the fluid-percussion model in cats. Am J Forensic Med Pathol 1998;19:129-36.

20.Zanier ER, Lee SM, Vespa PM, Giza CC, Hovda DA. Increased hippocampal CA3 vulnerability to low-level kainic acid following lateral fluid percussion injury. J Neurotrauma 2003;20:409-20.

21. Ginsberg MD, Sternau LL, Globus MY, Dietrich WD, Busto R. Therapeutic modulation of brain temperature: relevance to ischemic brain injury. Cerebrovasc Brain Metab Rev 1992;4:189-225.

22. Borer KT, Bestervelt LL, Mannheim M, Brosamer MB, Thompson M, Swamy U, et al. Stimulation by voluntary exercise of adrenal glucocorticoid secretion in mature female hamsters. Physiol Behav 1992;51:713-8.

23. Smith-Swintosky VL, Pettigrew LC, Sapolsky RM, Phares C, Craddock SD, Brooke SM, et al. Metyrapone, an inhibitor of glucocorticoid production, reduces brain injury induced by focal and global ischemia and seizures. J Cereb Blood Flow Metab 1996;16:585-98.

24. DeVries AC, Joh HD, Bernard O, Hattori K, Hurn PD, Traystman RJ, et al. Social stress exacerbates stroke outcome by suppressing Bcl-2 expression. Proc Natl Acad Sci U S A 2001;98:11824-8.

25. Vanderwolf $\mathrm{CH}$, Cain DP. The behavioral neurobiology of learning and memory: a conceptual reorientation. Brain Res Brain Res Rev 1994;19:264-97.

26. Qu M, Mittmann T, Luhmann HJ, Schleicher A, Zilles K. Long-term changes of ionotropic glutamate and GABA receptors after unilateral permanent focal cerebral ischemia in the mouse brain. Neuroscience 1998; 85:29-43.

27. Griesbach GS, Hovda DA, Molteni R, Wu A, GomezPinilla F. Voluntary exercise following traumatic brain injury: brain-derived neurotrophic factor upregulation and recovery of function. Neuroscience 2004;125: 129-39.

28. Hoffman-Goetz L, Spagnuolo PA, Guan J. Repeated exercise in mice alters expression of IL-10 and TNFalpha in intestinal lymphocytes. Brain Behav Immun 2008;22:195-9.

29. McEwen BS, Magarinos AM. Stress and hippocampal plasticity: implications for the pathophysiology of affective disorders. Hum Psychopharmacol 2001;16(S1): S7-S19.

30. Hansson AC, Sommer WH, Metsis M, Stromberg I, Agnati LF, Fuxe K. Corticosterone actions on the hippocampal brain-derived neurotrophic factor expression are mediated by exon IV promoter. J Neuroendocrinol 2006;18:104-14. 